\title{
The Longitude Dependence of the Dayside F Region Trough: A Detailed Model-Observation Comparison
}

\author{
J. J. SoJKa And R. W. Schunk \\ Center for Atmospheric and Space Sciences, Utah State University, Logan
}

J. A. Whalen

Geophysics Laboratory, Hanscom Air Force Base, Massachusetts

\begin{abstract}
The nighttime main $F$ region trough extends into the sunlit afternoon sector. This trough feature exhibits both a strong magnetic activity dependence and a longitude (UT) dependence. Whalen (1987), using International Geophysical Year (IGY) ionosonde data, showed that both of these effects are readily extracted from $f_{o} F_{2}$ observations. In this study we show that the longitude effect is the same as that contained in the Utah State University time-dependent ionospheric model. It arises from the offset of the geomagnetic axis from the geographic axis. The magnetic activity dependency is associated with the westward convection in the afternoon sector. It is also contained in the ionospheric model via the empirical magnetospheric convection model. This oneto-one observational-model agreement is unique; to date no other magnetic activity dependent ionospheric feature has been simulated this systematically.
\end{abstract}

\section{INTRODUCtion}

The $F$ region ionosphere responds on spatial scale sizes ranging from meters to thousands of kilometers to changes in geomagnetic activity. At most scale sizes the ionospheric activity dependencies are only known statistically. On the smaller, subkilometer, irregularity scale size these dependences are regarded as "turbulent" and, hence, only predictable in a statistical sense [Tsunoda, 1988]. At larger scales, the $F$ region depends directly on magnetospheric convection (electric field) and precipitation (aurora) and these, in turn, depend upon magnetic activity. The ionospheric density is known to exhibit both positive and negative phase responses on a global scale to major magnetospheric storms. However, as well-documented as these effects are, they are still extremely difficult to predict in a quantitative manner.

The purpose of this paper is to show that the ionospheric $F$ region trough feature analyzed by Whalen $[1987,1989 a$, b] is contained in the Utah State University (USU) timedependent ionospheric model [cf. Schunk, 1988, and references therein]. This feature is the extension of the midlatitude $F$ region trough into sunlight in the afternoon section. In this region, low density plasma is convected westward (sunward) to produce a depleted dayside trough. The magnitude and morphology of this trough are highly dependent on magnetic activity [Whalen, 1987, 1989b]. Since the dependence arises from magnetospheric convection, it is built into the USU ionospheric model in so far as empirical convection electric fields can represent the true magnetospheric convection. Whalen also showed that this trough feature has a unique longitude dependence arising from the offset between the geographic and geomagnetic poles. Such a longitude effect is also present in the USU ionospheric model.

Copyright 1990 by the American Geophysical Union.

Paper number 90JA00636.

0148-0227/90/90JA-00636\$02.00

\section{Leading Edge of Dayside Trough (LEDT)/ \\ Trailing Edge of Nightside Trough (TENT)}

Whalen $[1987,1989 a, b]$ showed that the high and midlatitude ionosonde database from the IGY period contains unique $F$ region trough observations. Using these data he showed the mid-latitude trough as an instantaneous pattern that depends on magnetic activity conditions. This magnetic activity dependence was also found to be longitude dependent. Figure 1 (from Whalen [1989a]) shows an $f_{o} F_{2}$ diurnal variation as measured by a typical northern hemisphere ionosonde station. The solid curve represents the quiet day $f_{o} F_{2}$ signature with a well-defined daytime maximum density. In comparison, the disturbed day shows a much different daytime signature. The shaded region of low density plasma is referred to as a "bite-out" by Whalen [1987].

The size of this feature was shown to be longitude and magnetic activity dependent by Whalen [1987]. As magnetic activity increased, the shaded region extended to earlier local times because the disturbed day "bite-out" would occur earlier. By contrasting this site with others at a similar longitude, Whalen showed that the LT of this disturbed day "bite-out" mapped into a spatial picture of the mid-latitude trough. In fact, the sharp drop in density on the disturbed day in Figure 1 corresponded to the leading edge of the daytime trough (LEDT). With increasing activity, this trough edge moved to earlier local times as well as to lower latitudes. In some longitude sectors, the LEDT did not appear to have a strong magnetic activity dependence. However, in such sectors other features, i.e., the trailing edge of the nighttime trough (TENT), showed a magnetic activity dependence.

The USU ionospheric model exhibits longitudinal dependencies, formally referred to as UT dependencies. These UT dependencies include marked trough variability during disturbed as well as quiet geomagnetic activity [Sojka et $a l ., 1981 a, 1981 b]$. Whalen chose to study the LEDT and he identified its location and extent in a snapshot form by reconstructing data from many ionosondes. Our iono- 


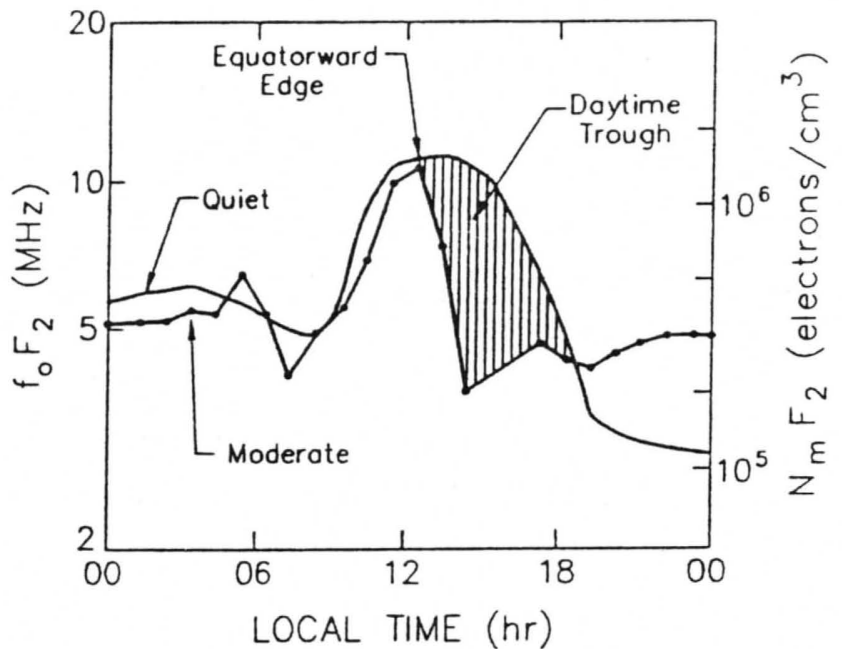

Fig. 1. The daytime trough in local time. Diurnal variation of $N_{m} F_{2}$ for 2 days recorded at Troms $\varnothing$, a station in the Scandinavian IGY ionosonde chain. The daytime trough appears as the "depletion" in ionization (shaded) that occurs on the moderately disturbed day. The "depletion" appears because of the equatorward movement of the trough during geomagnetic activity (from Whalen $[1989 a])$.

spheric model was run with appropriate parameters so that we could compare the predicted trough characteristics with the ionosonde data. This run took 2.5 hours of CPU time on a Cray XMP computer. The model uses an offset tilteddipole magnetic field, this is the source of the longitudinal effects. The specific solar, geomagnetic, and seasonal conditions were chosen to be consistent with the Whalen [1987, $1989 a, b]$ studies, namely, solar maximum (1981), early winter (November 22), and moderate activity ( $K p=2$ to 3 ). For this particular study, a Heppner-Maynard (IMF southward with $B y$ negative) convection pattern was used [Heppner and Maynard, 1987]. This convection pattern has a polar cap potential of $76 \mathrm{kV}$. The $K p$ index affects the simulation in distinct ways: first, the polar cap potential scales linearly with $K p$; second, the auroral precipitation increases as $K p$ increases (the Hardy statistical oval is used [Hardy et al., 1987]); third, both these two patterns expand equatorward as $K p$ increases; and fourth, the neutral atmosphere density and composition is changed since the Mass spectrometer and incoherent scatter (MSIS) statistical model depends on magnetic activity [Hedin, 1987].

Figure 2 shows six snapshots at 4-hour UT intervals beginning at $0100 \mathrm{UT}$. These are polar dial distributions of $N_{m} F_{2}$ grey scaled over the latitude range from $50^{\circ}$ to the pole in the northern hemisphere. From Figure 2 it is evident that each UT is somewhat different. The mid-latitude trough, a light-shaded region extending from the afternoon sector through midnight into the morning sector, is present in each panel. Lines have been superimposed on these diagrams to denote the leading edge (LEDT) and the trailing edge (TENT) of the trough. For both boundaries, two lines have been drawn at specific densities $\left(1 \times 10^{5}\right.$ and $3.5 \times 10^{5}$ $\left.\mathrm{cm}^{-3}\right)$. At both 0100 and $2100 \mathrm{UT}$, these two lines are very close together in the LEDT, i.e., the early afternoon sector where a corotating ground station first encounters the
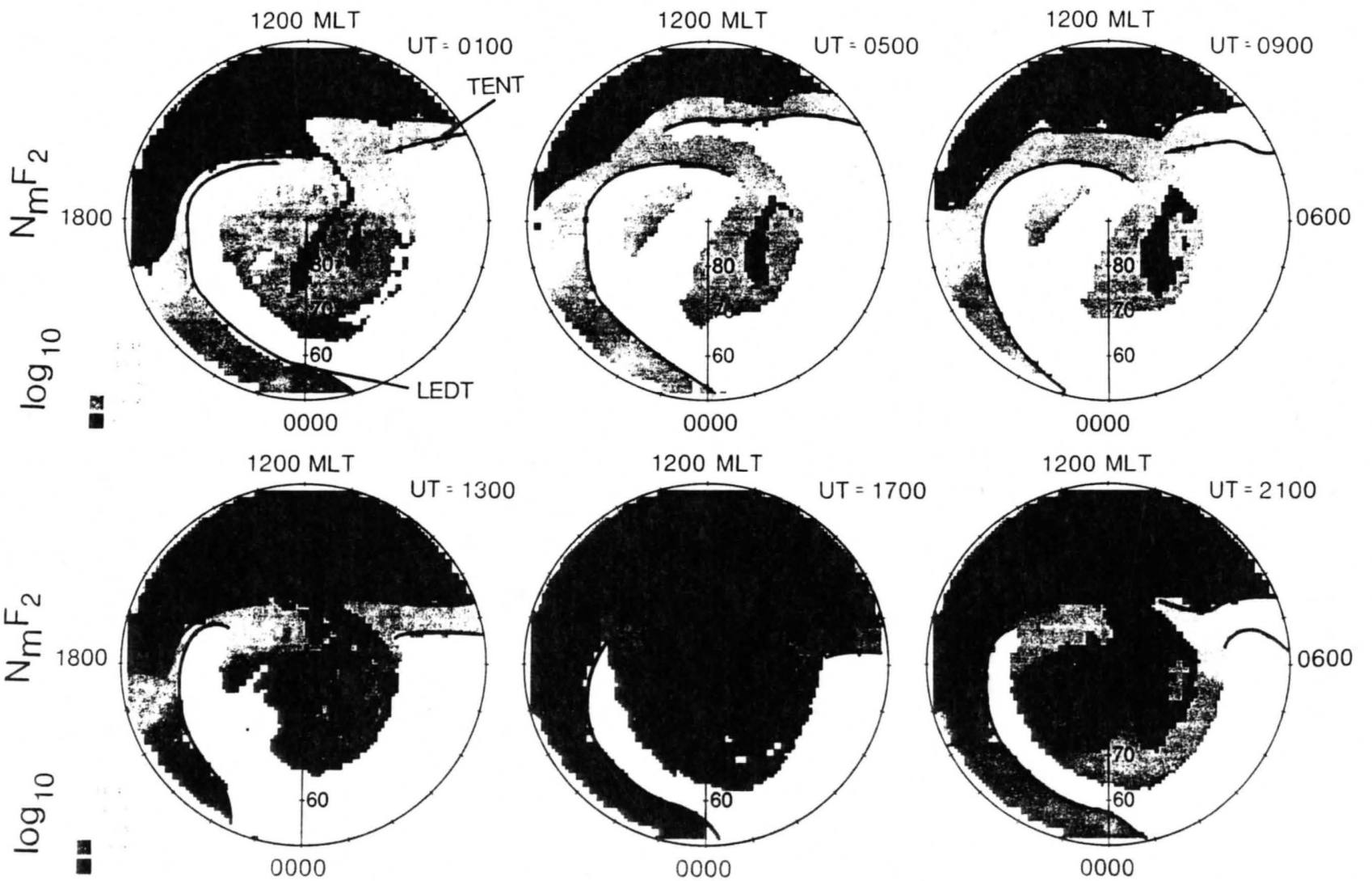

Fig. 2. Model $N_{m} F_{2}$ values at six universal times. Each panel shows grey scaled densities in a magnetic latitudeMLT polar plot. The darkest shading represents the largest densities $\left(>10^{6} \mathrm{~cm}^{-3}\right)$, while white represents the lowest densities $\left(<4 \times 10^{4} \mathrm{~cm}^{-3}\right)$. 
mid-latitude trough. At these UTs the transition from high daytime densities to low trough densities is extremely rapid ( $\lesssim 30 \mathrm{~min}$ ). In contrast, at 0500 and $0900 \mathrm{UT}$ the density decrease at the LEDT boundary is very slow (several hours) and quite complex. This UT (longitude) dependence in the model predicts that as magnetic activity increases the LEDT feature would, for a given ionosonde site, move to earlier local times and be observed at lower latitudes. Both of these trends are consistent with the Whalen $[1987,1989 a, b]$ analysis of IGY data.

The signatures of the afternoon sector LEDT and the prenoon TENT are ambiguous at certain UTs. At both 0500 and $0900 \mathrm{UT}$, the $10^{5} \mathrm{~cm}^{-3}$ LEDT line extends into the prenoon sector, while at 0500 UT the $10^{5} \mathrm{~cm}^{-3}$ TENT line extends into the afternoon sector. This ambiguity would lead to ionosonde sites at different latitudes seeing different diurnal patterns even though their longitudes are similar. These model results are also consistent with the Whalen IGY analysis.

To avoid further confusion in terminology, the model UT dependence will be referred to as a "longitudinal dependence," since it is the same phenomenon as that discussed by Whalen.

\section{Model Longitude Dependence}

The series of six snapshots in Figure 2 indicate that the LEDT is, in the magnetic frame, somewhat static. In other words, the $10^{5} \mathrm{~cm}^{-3}$ line has the same shape and location in all six cases. This is not the case for the trailing edge of the nighttime trough (TENT) in the dawn sector, which is seen to move in MLT. Figure 3 shows only the $10^{5} \mathrm{~cm}^{-3}$ lines representing the LEDT and TENT boundaries in the magnetic frame at 12 separate, but equally spaced, UTs. The 12 LEDT lines overlap each other and trace a single boundary; however, the dayside extent depends upon longitude (UT). In sharp contrast, the TENT boundary lines exhibit a marked variability through the 0600 to 1200 MLT

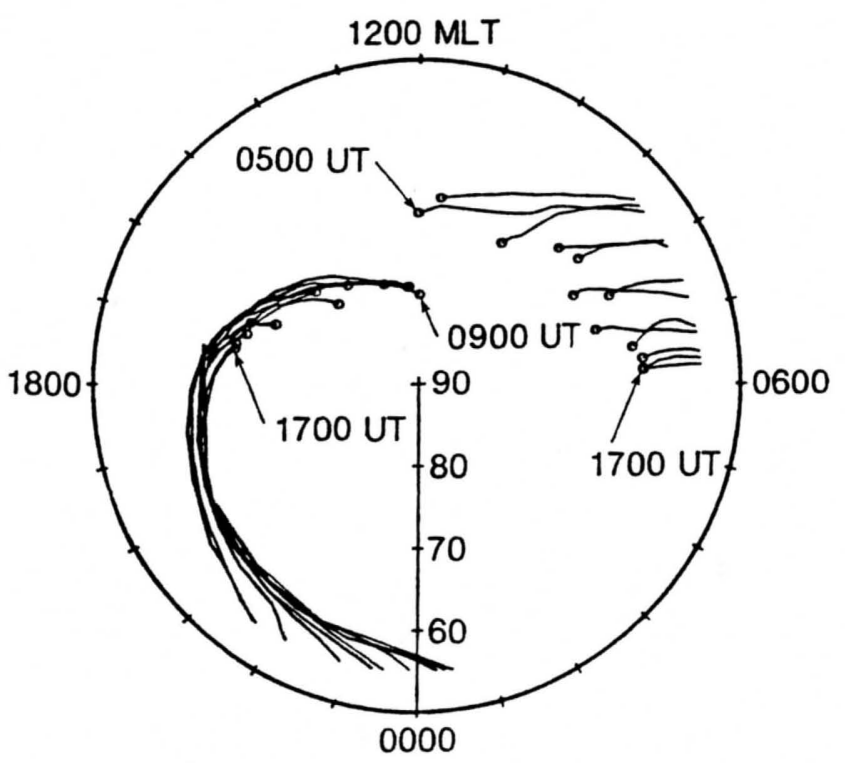

Fig. 3. Locations of the modeled LEDT (dusk sector) and TENT (dawn sector) boundaries for 12 different UTs plotted in a magnetic latitude-MLT polar plot. The LEDT and TENT are defined by the $10^{5} \mathrm{~cm}^{-3}$ density threshold at the equatorward edge of the trough. Only the extreme UTs are labeled on the LEDT and TENT curves. sector. This variability is associated with the motion of the solar terminator in the magnetic frame.

Figure 4 demonstrates the solar control of the TENT boundary; the lines in Figure 3 are replotted in the geographic latitude-LT frame. Now, the dawn sector (TENT) boundary falls along the solar terminator, which is a line along the dawn-dusk direction and is indicated by the dashed line in Figure 4. In the geographic frame, the LEDT boundary is now extremely variable, extending from below $50^{\circ}$ up to $90^{\circ}$ geographic latitude and from 0800 to $2400 \mathrm{LT}$. It is this latter variability which for different ground sites (geographic locations) leads to the Whalen LEDT observation.

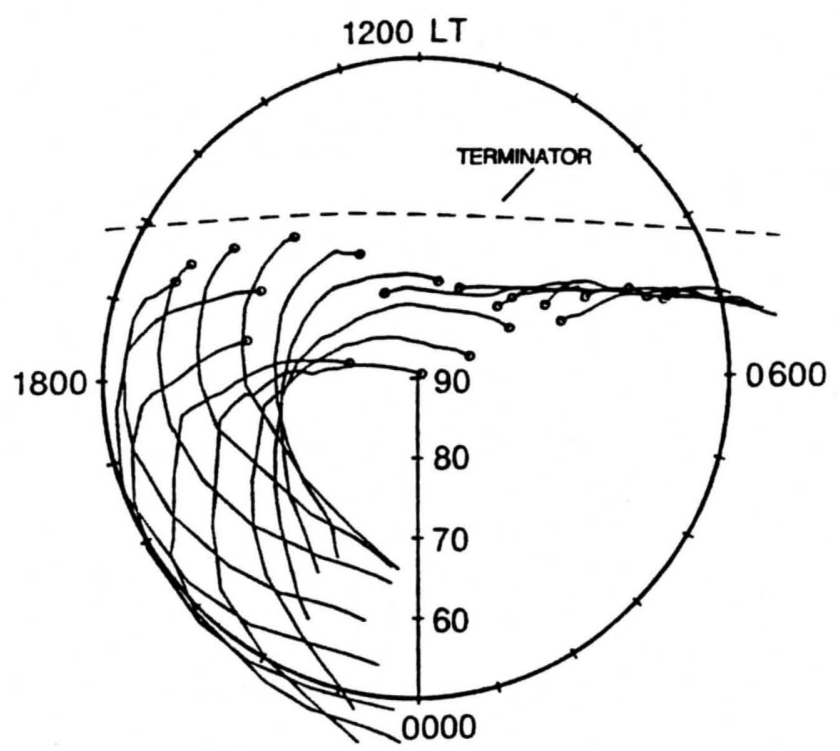

Fig. 4. The same LEDT and TENT boundaries as in Figure 3 plotted in a geographic latitude-LT polar plot. The dashed line indicates the solar terminator.

Both Figures 3 and 4 show boundaries defined by the $N_{m} F_{2}$ density falling below $10^{5} \mathrm{~cm}^{-3}$. This does not represent the same criterion used by Whalen $[1987,1989 a, b]$ to deduce the LEDT. The trends shown in these two figures are, however, representative of the longitudinal (UT) dependence in the ionospheric model. The specific shape of the LEDT boundary (Figure 3) would vary with convection pattern and level of magnetic activity.

\section{COMPARISON OF THE EMPIRICAL AND THEORETICAL LEDT}

Whalen [1987] showed that a well-defined afternoon sector "bite-out" was observed at all stations within a specific longitude range $\left(250^{\circ} \mathrm{E}\right.$ to $\left.75^{\circ} \mathrm{E}\right)$. Sites outside this range would not always see an afternoon sector "bite-out", although they may see a morning sector "bite-out". Figure 5 shows, in a geographic polar plot, the distribution of sites (denoted by + ) which saw the "bite-out" in the afternoon sector. The line around these sites represents Whalen's envelope of latitudes and longitudes for which the LEDT would be detectable over a wide range of magnetic activity levels in the IGY database. Sites outside this envelope would see the "bite-out" only on occasions. An example of one such site is the cross labeled BW (Barrow), which is included in a more detailed comparison later in this section. The other labeled crosses are also discussed later in this section. 


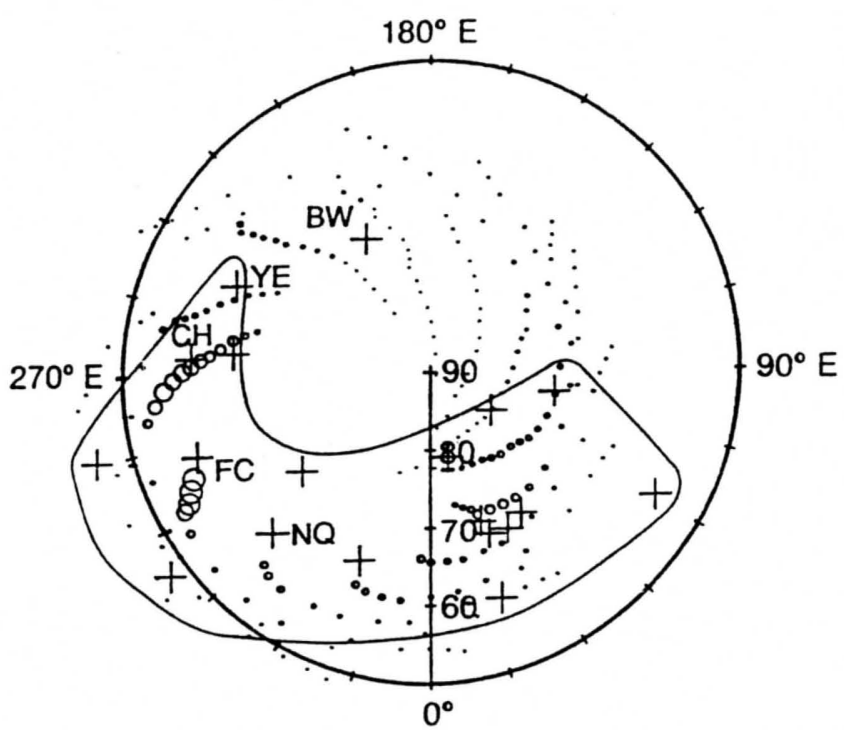

Fig. 5. A geographic polar plot of the observed "bite-out" (crosses) and model LEDT boundaries at 12 different UTs (circles). The diameter of the circle represents the steepness of the LEDT density gradient. The closed loop represents Whalen's empirical envelope of sites from which the LEDT "bite-out" can be observed.

Superimposed on Figure 5 are 12 sets of model LEDT trough boundaries. These correspond to the lines shown in Figures 3 and 4 as the $10^{5} \mathrm{~cm}^{-3}$ trough boundaries. All longitudes see such a boundary. However, whether it appears as a "bite-out" or not depends on both the local time at which it is seen and the steepness of the LEDT density gradient. In Figure 5 the steepness of the density gradient is represented by the diameter of the plotted circles. Regions where a sharp density LEDT is observed are regions of open circles, while regions of a very gradual density fall off are effectively points. The region of open circles falls inside the Whalen envelope for the "bite-out" phenomenon. This distribution of open circles is representative of the model output for winter conditions for a wide range of geomagnetically active conditions. The specific distribution of large and small circles inside the Whalen envelope would be convection model dependent. The site labeled BW in Figure 5 lies in a region where the ionospheric model predicts a weak "bite-out" signature. Hence, although it lies outside the observation envelope defined by Whalen, it is still consistent with the region where the "bite-out" phenomenon is expected to be theoretically operating.

The magnetic activity dependence of the LEDT is shown in Figure 6 as a set of four dashed lines, each labeled with the appropriate magnetic activity level. These data are from the Whalen $[1987,1989 a]$ studies. As magnetic activity increases, the LEDT moves to earlier local times and to lower magnetic latitudes. Superimposed upon this plot are the model curves for the LEDT (solid lines). These are the same curves shown in the previous figures that represent the $10^{5}$ $\mathrm{cm}^{-3}$ threshold density. The model was run for a specific day for which the magnetic activity level ranged from a $K p$ of 2 to 3 . The model results are generally consistent with the statistical trends deduced by Whalen. In the immediate postnoon sector, the model LEDT curve appears to corre-

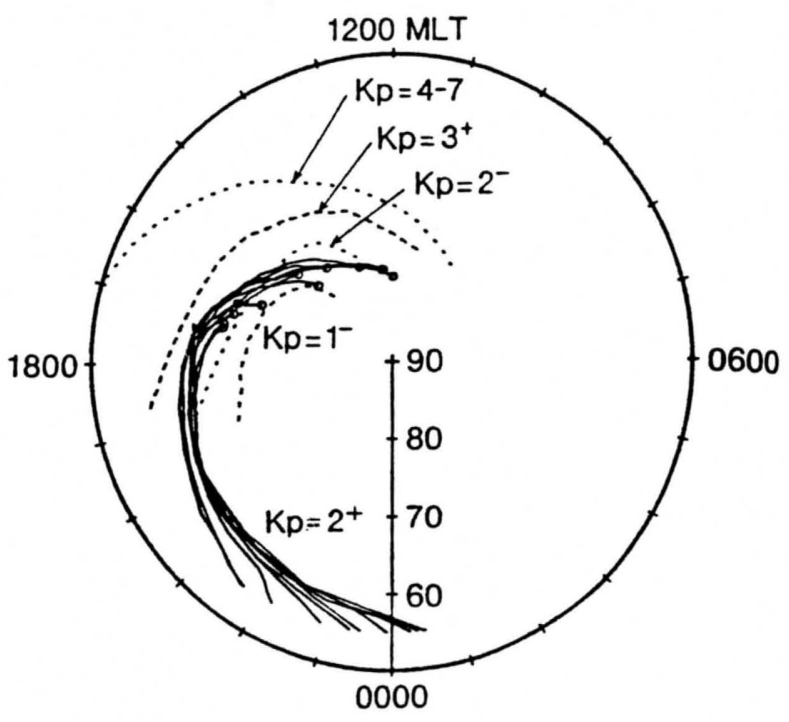

Fig. 6. LEDT boundaries from Whalen's IGY analysis (dashed lines) and from the ionospheric model (solid lines) in a magnetic latitude-MLT polar plot. Each curve is labeled with the appropriate geomagnetic activity level.

spond to a lower magnetic activity level when compared to the measurements. This discrepancy is associated with the specific convection pattern used in the model study. The convection pattern used was a southward IMF pattern with a strong negative $B y$ component. Other patterns would result in the LEDT curves having somewhat modified shapes. Indeed, Whalen [1989b] showed that when 31 days of data from the IGY period were analyzed the spread in shape of the LEDT curves became complex. With the additional 31 days of data, the four simple dashed lines in Figure 6 would become significantly more spread out; i.e., the dashed line would only represent a mean LEDT curve for a particular level of magnetic activity.

Figure 7 compares the modeled and observed $N_{m} F_{2}$ densities for five sites. Of particular note is the observed quiet day (light line) and observed disturbed day (heavy line) difference. The region referred to as the "bite-out" is shaded. This region increases in LT extent as one moves from station to station in an eastward direction. The model results, shown as the line with circles, follow almost exactly this bite-out LT trend. Barrow (top panel) is the site that lies outside the Whalen envelope (see Figure 5). It shows a small "bite-out", which on this day was discernible. The model calculation for this site agrees well with this small "biteout". All four of the remaining sites in Figure 7 lie within the Whalen envelope in Figure 5. In each case, the general "bite-out" feature is present in the model results. Each model value has a 2-hour UT spread due to the binning of the model densities in UT. This can introduce relative LT shifts of \pm 1 hour between the model and observation. However, the absolute densities do not agree exactly, since the convection pattern adopted in the model calculation is only a representative empirical pattern. Indeed, it is extremely difficult to verify what the convection pattern was on a specific day during the IGY period. Qualitatively, the trends are found to be the same, which is what we wanted to show in this study. 
23 December 1958
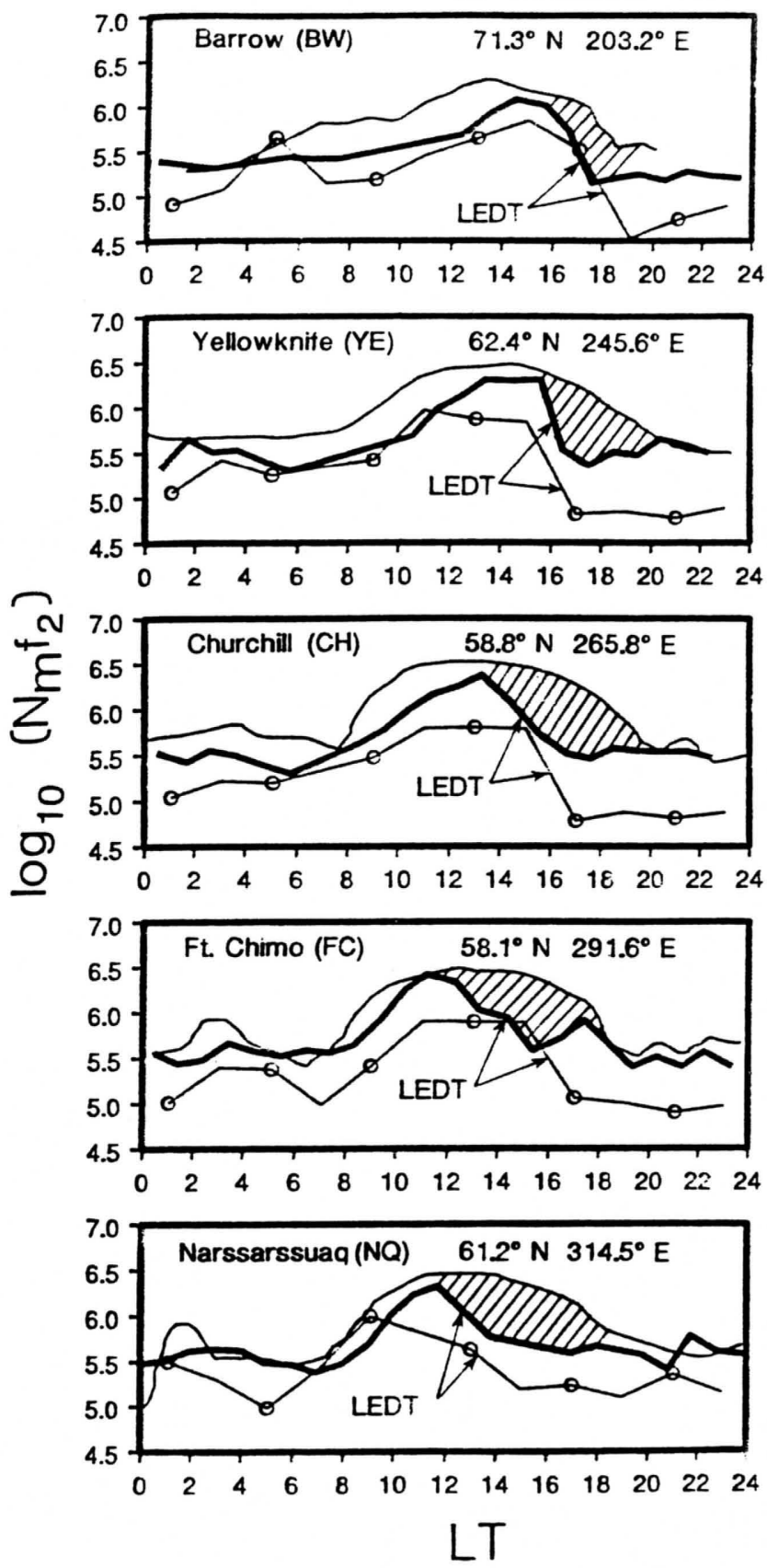

Fig. 7. Diurnal variation of $N_{m} F_{2}$ for five high latitude sites. The observed quiet day curve (light line), observed disturbed day curve (heavy line), and modeled disturbed day curve (light line with circles) are superimposed in each panel. The shading highlights the "bite-out" feature.

\section{Discussion and Summary}

The $F$ region density dependence on geomagnetic activity is well documented; however, it is not well understood. Theoretically, the individual mechanisms which cause the density variations (temporal and spatial variations in the magnetospheric electric field, auroral precipitation, neutral atmosphere, and neutral wind) are well defined. In a composite sense their relative interdependencies are not well defined. Ionospheric models do produce disturbed $F$ region densities, but the specific results depend sensitively on the magnetospheric and thermospheric inputs to the model [ $\mathrm{So}$ $j k a$ and Schunk, 1983, 1984].

The analysis of Whalen [1987, 1989a, $b]$ focused on one particular $F$ region activity dependence. In this paper a representative model study has been compared with the empirical findings of Whalen. Morphologically, the longitude dependence, the latitude dependence, and the magnetic activity dependence of the LEDT "bite-out" observed by the IGY ionosonde stations can be theoretically generated. This feature, observed in the American and European high latitude sectors, is shown to be a sensitive indicator of the status of ionospheric activity. The dependence is primarily associated with the variability of magnetospheric electric field convection of low density plasma westward, into sunlight, in the afternoon sector.

With present day interests and requirements in both realtime and predictive ionospheric modeling of a meteorological rather than climatological nature [Schunk and Sojka, 1987], this observable ionospheric feature has great potential. Each site within the "bite-out" sector in Figure 5 can readily be used to deduce the level of geomagnetic activity as the LEDT boundary moves across the station. A distribution of sites can be used to deduce the temporal variation of the magnetic activity over a 10-hour period each day. Also, observing sites at longitudes outside this longitude sector could be used to extend the coverage by focusing on the TENT. These empirical results would be a direct test of real-time or prediction models during active geomagnetic periods. They would also enable a direct determination of the ionospheric activity level, in contrast to monitors such as $a p$ and $K p$ which are indirect and rarely available in real time. Work is currently under way to use this ionospheric phenomenon in ionospheric specification modeling.

Acknowledgments. This research was supported by NASA grant NAGW-1547, AFOSR contract F49620-86-C-0109, and AFOSR grant AFOSR-90-0026 DEF to Utah State University.

The editor thanks W. B. Hanson and D. G. Torr for their assistance in evaluating this paper.

\section{REFERENCES}

Hardy, D. A., M. S. Gussenhoven, R. Raistrick, and W. J. McNeil, Statistical and functional representations of the pattern of auroral energy flux, number flux, and conductivity, J. Geophys. Res., 92, 12,275-12,294, 1987.

Hedin, A. E., MSIS-86 thermospheric model, J. Geophys. Res., 92, 4649-4662, 1987.

Heppner, J. P., and N. C. Maynard, Empirical high-latitude electric field models, J. Geophys. Res., 92, 4467-4489, 1987.

Schunk, R. W., A mathematical model of the middle and high latitude ionosphere, Pure Appl. Geophys., 127, 255-303, 1988.

Schunk, R. W., and J. J. Sojka, Numerical simulations of the ionosphere: Climatology and weather, EOS Trans. AGU, 69, 153-164, 1987.

Sojka, J. J., and R. W. Schunk, A theoretical study of the highlatitude $F$ region's response to magnetospheric storm inputs, J. Geophys. Res., 88, 2112-2122, 1983.

Sojka, J. J., and R. W. Schunk, A theoretical $F$ region study of ion compositional and temperature variations in response to magnetospheric storm inputs, J. Geophys. Res., 89, 23482358, 1984.

Sojka, J. J., W. J. Raitt, and R. W. Schunk, A theoretical study of the high-latitude winter $F$ region at solar minimum for low magnetic activity, J. Geophys. Res., 86, 609-621, $1981 a$.

Sojka, J. J., W. J. Raitt, and R. W. Schunk, Plasma density 
features associated with strong convection in the winter highlatitude $F$ region, J. Geophys. Res., 86, 6908-6916, $1981 b$.

Tsunoda, R. T., High-latitude $F$ region irregularities: A review and synthesis, Rev. Geophys., 26, 719-760, 1988.

Whalen, J. A., Daytime $F$ layer trough observed on a macroscopic scale, J. Geophys. Res., 92, 2571-2576, 1987.

Whalen, J. A., The daytime $F$ layer trough, in 1988 Cambridge Workshop Proceedings on Polar Cap Dynamics and High-Latitude Ionospheric Turbulence, edited by T. Chang, G. B. Craw, and J. R. Jasperse, p. 183, Scientific Publishers, Massachusetts, $1989 a$.

Whalen, J. A., The daytime $F$ layer trough and its relation to ionospheric-magnetospheric convection, J. Geophys. Res., 94, $17,169-17,184,1989 b$.

R. W. Schunk and J. J. Sojka, Center for Atmospheric and Space Sciences, Utah State University, Logan, UT 84322.

J. A. Whalen, Geophysics Laboratory, Hanscom Air Force Base, Bedford, MA 01731.

(Received November 2, 1989; revised March 5, 1990; accepted March 5, 1990.) 